\title{
Protein repertoire impact of Ubiquitin-Proteasome System impairment: Insight into the protective role of beta-estradiol
}

\author{
Annamaria D’Alessandro ${ }^{a, b}$, Simona D'Aguanno ${ }^{a, b}$, Maria Teresa Cencioni ${ }^{b}$, \\ Luisa Pieroni ${ }^{a, b}{ }^{\text {, Adamo Diamantini }}{ }^{b}$, Luca Battistini ${ }^{b}$, Patrizia Longone ${ }^{b}$, Alida Spalloni ${ }^{b}$, \\ Vincenzo De Laurenzi ${ }^{c}$, Sergio Bernardini ${ }^{a}$, Giorgio Federici ${ }^{a}, d$, Andrea Urbani ${ }^{a, b, *}$
}

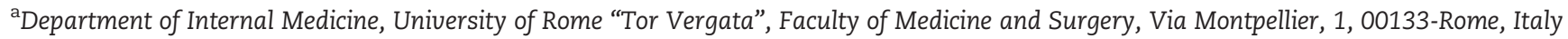

'banta Lucia Foundation-IRCCS, Via del Fosso di Fiorano, 64, 00143-Rome, Italy

"Department of Biomedical Science, University" G. D’Annunzio", Via dei Vestini 31, 66013-Chieti, Italy

${ }^{d}$ Children's Hospital “Bambino Gesù”-IRCCS, Piazza Sant'Onofrio, 4, 00165-Rome, Vatican City State

\section{A R T I C L E I N F O}

Article history:

Received 11 August 2011

Accepted 14 November 2011

Available online 2 December 2011

Keywords:

Ubiquitin-Proteasome System

impairment

Neurodegenerative Diseases

2-Dimensional Electrophoresis

separation

Shotgun Proteomics

Meta-analysis

beta-Estradiol

\begin{abstract}
A B S T R A C T
The Ubiquitin-Proteasome System (UPS) and the Autophagy-Lysosome Pathways (ALP) are key mechanisms for cellular homeostasis sustenance and protein clearance. A wide number of Neurodegenerative Diseases (NDs) are tied with UPS impairment and have been also described as proteinopathies caused by aggregate-prone proteins, not efficiently removed by proteasome. Despite the large knowledge on proteasome biological role, molecular mechanisms associated with its impairment are still blur. We have pursued a comprehensive proteomic investigation to evaluate the phenotypic rearrangements in protein repertoires associated with a UPS blockage. Different functional proteomic approaches have been employed to tackle UPS impairment impact on human NeuroBlastoma (NB) cell lines responsive to proteasome inhibition by Epoxomicin. 2Dimensional Electrophoresis (2-DE) separation combined with Mass Spectrometry and Shotgun Proteomics experiments have been employed to design a thorough picture of protein profile. Unsupervised meta-analysis of the collected proteomic data revealed that all the identified proteins relate each other in a functional network centered on beta-estradiol. Moreover we showed that treatment of cells with beta-estradiol resulted in aggregate removal and increased cell survival due to activation of the autophagic pathway. Our data may provide the molecular basis for the use of beta-estradiol in neurodegenerative disorders by induction of protein aggregate removal.
\end{abstract}

(c) 2011 Elsevier B.V. All rights reserved.

\footnotetext{
Abbreviations: 2-DE, 2-Dimensional Electrophoresis; 7-ADD, 7-Amino-actinomycin; ALP, Autophagy-Lysosome Pathways; ALS, Amyotrophic Lateral Sclerosis; AV, Annexin V; BEH, Bridged Ethyl Hybrid; CMA, Chaperone-Mediated Autophagy; ECL, Enhanced ChemiLuminescence; EMRT, Exact Mass Retention Time; ESI-Q-TOF, Electrospray-Quadrupole-Time Of Flight; FACS, Fluorescence Activated Cell Sorting; HCCA, $\alpha$-Cyano-4-HydroxyCinnamic Acid; HSPs, Heat Shock Proteins; HSP10 (60, 70, 90, etc.), Heat Shock Protein 10 kDa (60, 70 , 90, etc.); IEF, IsoElectroFocusing; IPA, Ingenuity Pathways Analysis; IPKB, Ingenuity Pathways Knowledge Base; LC3, microtubule-associated protein 1 Light Chain 3; LC-MS/MS, Liquid Chromatography coupled with tandem MS; LC-MS ${ }^{\mathrm{E}}$, LC-MS using simultaneous acquisition of exact mass at high and low collision energy; MALDI-TOF, Matrix-Assisted Laser Desorption/Ionization; MPRs, Mannose 6-Phosphate Receptors; MS-PMF, Mass Spectrometry-Peptide Mass Fingerprinting; Mr, Molecular ratio; NB, NeuroBlastoma; NDs, Neurodegenerative Diseases; NIH, National Institutes of Health; Op18, Oncoprotein 18; PANTHER, Protein ANalysis THrough Evolutionary Relationships; PD, Parkinson's disease; pI, Isoelectric point; PI, Propidium Iodide; PIs, Proteasome Inhibitors; PLGS, ProteinLynx Global SERVER; ROI, Region of Interest; RP, Reversed Phase; TCA, TriChloroacetic acid; TIP47, Tail Interacting Protein47-kDa; UPS, Ubiquitin-Proteasome System; VGF, VGF nerve growth factor inducible protein

* Corresponding author at: Proteomic and Metabonomic Laboratory, CERC Fondazione, Santa Lucia, Via del Fosso di Fiorano 64, 00143 Rome, Italy. Tel.: +39 06501703215; fax: +3906501703332.

E-mail address: andrea.urbani@uniroma2.it (A. Urbani).
} 


\section{Introduction}

Many late-onset NDs are described as proteinopathies in which the accumulation of aggregate-prone proteins, dependent both on altered protein expression levels and on their physiological half-life, is the main distinctive feature of these diseases. While the causative role of protein aggregates is still matter of debate it seems clear that at least in some cases removal of aggregates is beneficial to cells. In eukaryotic cells the two main routes of protein clearance are represented by the UPS and ALP that are involved in regulatory mechanisms and in the adaptation to adverse environments [1]. Since UPS is more efficient than basal levels of macroautophagy, in the biological systems that have access to both pathways, proteasomes result to be the favored and dominating clearance route [2]. These scavenger systems target different proteins for degradation, such as proteins involved in the cell-cycle and abnormal proteins that, resulting from oxidative stress, disrupt normal cellular homeostasis [3,4]. Perturbations in both of these systems have been observed as cause or consequence in the pathogenesis of NDs $[5,6]$.

In order to elucidate the molecular changes occurring in proteinopathies we planned to perform a detailed proteomic analysis of a simple cellular model. We induced protein accumulation and aggregate formation in a neuroblastoma cell line by proteasome blockage. Proteasome Inhibitors (PIs) have already been used to develop neurodegenerative disease in animal models [7] as well as in cell culture models where these drugs induce the formation of peri-nuclear ubiquitinated inclusions [8]. A variety of compounds, both natural products and synthetic analogous, have been found to inhibit protein degradation by proteasome blockage [9]. Among these, Epoxomicin, a cell permeable, natural and irreversible proteasome inhibitor, has been used extensively as Parkinson's Disease (PD)-inducing toxins [10] in different models [7,10,11]. Here we have evaluated the biological effects of low-dose Epoxomicin treatment and its impact on the phenotype of a human NB cell line, described as an excellent model to study NDs [12]. In particular by different functional proteomic approaches we have clarified Epoxomicin rebounds on NB cells characterizing proteomics data flow through protein networks. Interestingly from proteomics data we have extrapolated the functional role of betaestradiol in Epoxomicin exposed cells. Moreover we have shown that estrogen treatment results in increased cell viability and the induction of autophagic processes to remove ubiquitinated inclusions. These findings suggest a novel mechanism through which $\beta$-estradiol can induce the removal of protein aggregates and thus its potential therapeutical use.

\section{Materials and methods}

\subsection{Materials}

Cell culture media, sera and additives were purchased from Invitrogen. Penicillin/streptomycin was obtained from Euroclone Life Sciences. Epoxomicin, $\beta$-estradiol, reagents used in vitality assays, materials for SDS/PAGE or Sodium Thiosulphate/PAGE and TCA protein determination reagent were from Sigma
Aldrich. Materials used for IEF, immunoblot experiments and ECL reagents were from Amersham GE Healthcare. Reagents used as viability probe and cell dye in FACS were from BD Pharmingen ${ }^{\mathrm{TM}}$. Reagents used in immunofluorescence experiments, antibodies against ubiquitin and $\beta$-actin were from Sigma Aldrich. Pro-Long Gold Antifade reagent was obtained from Invitrogen. Antibodies against p53, HSP60, ubiquitin, p21, caspase-8, HSP70, Op18 and TIP47 were from Santa Cruz Biotechnology while the antibody against LC3 was from Medical \& Biological Laboratories (MBL). Antibody antiVGF was kindly provided by Prof. Roberta Possenti. Organic solvents used in Mass Spectrometry were purchased from Romil. Trypsin-Mass Spectrometry Grade was from Promega while ZipTip C18 pipetting tips were purchased from Millipore. C18 Nanoease Atlantis column and Symmetry C18 column were from Waters. PicoTip Emitter was purchased from New Objective. The calibration peptide mix was purchased from Bruker Daltonics while Enolase Saccharomyces cerevisiae digestion used as internal standard was from Waters.

\subsection{Cell lines}

Human Neuroblastoma (SH-SY5Y, IMR-32 and SK-NAS) cells were maintained in Dulbecco's modified Eagle's High glucose medium supplemented with 10\% Fetal Bovine Serum, $2 \mathrm{mM}$ L-glutamine, 1\% NEAA, 1\% Sodium Pyruvate, $10 \mathrm{mM}$ HEPES and antibiotics. Human Neuroblastoma LAN-5 cells were grown in RPMI medium supplemented with $20 \%$ Fetal Bovine Serum, 2 mM L-glutamine , 1\% NEAA, 1\% sodium pyruvate, $10 \mathrm{mM}$ HEPES and antibiotics.

\subsection{Viability assays}

SH-SY5Y cells were seeded at $500 \times 10^{3}$ cells per well in 6-well plates and exposed to different drugs. Epoxomicin and betaestradiol were dissolved respectively in DMSO and Ethanol, according to the manufacturer instructions. To obtain the desired concentration (30 nM Epoxomicin and $100 \mathrm{nM}$ beta-estradiol) both drugs were diluted in DMEM (1:1000, v/v). In one set of experiments employing Epoxomicin, cells were grown for 1 day and then exposed to Epoxomicin or left under resting condition. In the experiments employing beta-estradiol cells were grown for 1 day and then pre-incubated with beta-estradiol and treated with Epoxomicin.

In this investigation experimental conditions included basal condition, treatment with distinct concentrations of beta-estradiol and Epoxomicin for different time of exposure. Viable cells were quantified by counting the number of intact nuclei after incubation with Tripan blue. Experiments were performed in triplicate and repeated three times with similar results.

\subsection{Flow cytometric analysis}

$1 \times 10^{6} \mathrm{SH}$-SY5Y cells were suspended in binding buffer $\mathrm{pH} 7.4$ (10 mM Hepes, $140 \mathrm{mM} \mathrm{NaCl}$, and $2.5 \mathrm{mM} \mathrm{CaCl2}$ ) in which Fluorescein isothiocyanate (FITC)-annexin V $(50 \mu \mathrm{g} / \mathrm{ml}$, 10 min; RT) was diluted. Staining with Annexin V and 7Amino-actinomycin D (7-AAD; $\left.12.5 \mu \mathrm{g} / \mathrm{ml}, 10 \mathrm{~min} ; 4^{\circ} \mathrm{C}\right)$ was used as vital dyes that allow to discriminate early apoptotic 
cells from necrotic/late apoptotic cells [13]. To evaluate cell cycle distribution, sample preparation and analysis were performed with Propidium iodide (PI), as described by Kalejta RF et al [14]. Briefly, SH-SY5Y cells $\left(1 \times 10^{6}\right.$ cells $\left./ \mathrm{ml}\right)$ suspended in PBS were fixed with $70 \%$ ethanol (1:2, v/v; 2-3 days), washed and suspended in the staining solution $(1 \mathrm{mg} / \mathrm{ml} \mathrm{PI}, 0.1 \%$ RNase A/PBS; w/v). FACS analysis on both NB Annexin V and/or 7AAD and on PI stained cells was performed using a BD FACSCalibur system (Becton Dickinson) and a BD FACSCanto system, respectively. FlowJo analysis software was used. Each measurement was carried out several times with the same results.

\subsection{Immunoblotting}

Human Neuroblastoma cells (SH-SY5Y, LAN-5, IMR-32 and SK-NAS) were collected and lysed in O'Farrel modified buffer (7 M Urea, 2 M Thiourea, 50 mM DTT, 4\% CHAPS, 40 mM Tris $0.5 \% \mathrm{v} / \mathrm{v}$ ampholytes). Equal amount of soluble proteins $(60 \mu \mathrm{g})$, quantified by Trichloroacetic acid (TCA adapted procedure [15] by using Bobine serum Albumine (BSA) as standard, were loaded on polyacrylamide gels. In particular SDS-PAGE and Sodium Thiosulphate-PAGE were blotted on Hybond nitrocellulose membrane and incubated $\mathrm{O} / \mathrm{N}$ with the following primary antibodies: MAb anti-p53 (0.4 $\mu \mathrm{g} / \mathrm{ml}), \mathrm{Ab}$ anti-p21(0.8 $\mu \mathrm{g} /$ $\mathrm{ml}), \mathrm{Ab}$ anti-caspase-8 $(0.4 \mu \mathrm{g} / \mathrm{ml}), \mathrm{MAb}$ anti-ubiquitin $(0.2 \mu \mathrm{g} /$ $\mathrm{ml}), \mathrm{Ab}$ anti-TIP47 $(1 \mu \mathrm{g} / \mathrm{ml}), \mathrm{Ab}$ anti-HSP70 $(1 \mu \mathrm{g} / \mathrm{ml}), \mathrm{Ab}$ antiOp18 $(1 \mu \mathrm{g} / \mathrm{ml}), \quad \mathrm{Ab}$ anti-LC3 $(0.1 \mu \mathrm{g} / \mathrm{ml}), \mathrm{MAb}$ anti-HSP60 $(0.5 \mu \mathrm{g} / \mathrm{ml}), \mathrm{MAb}$ anti- $\beta$-actin $(0.2 \mu \mathrm{g} / \mathrm{ml})$ and Ab anti-VGF $(1 \mu \mathrm{g} /$ $\mathrm{ml})$. Blots were then probed with specific secondary antibodies conjugated with horseradish peroxidise and detected with Enhanced Chemiluminescence Plus reagent. Images were digitized on a flatbed scanner and the density of specific bands was normalized with those of $\beta$-actin. Relative quantization of specific bands was made up by using the NIH Image J software, developed at the National Institutes of Health (http:// rsb.info.nih.gov/ij). Statistical significance was determined by Student's t-test, using $\mathrm{p}<0.05$ as the significance level.

\subsection{2-Dimensional gel electrophoresis (2-DE)}

2D gel electrophoresis was carried out according to a modified Gorg A. protocol [16] on immobilized 3-10NL pH gradient strips $(18 \mathrm{~cm})$. Protein samples were precipitated in a mix of organic solvent and solubilized in O'Farrell modified buffer [17] prior analysis. A total of $100 \mu \mathrm{g}$ of protein extract were used for preparative gels for each condition: NB wild-type cells (untreated) and 24 and $48 \mathrm{~h}$ Epoxomicin treated NB cells (Epoxomicin 24 and 48 h), 2-DE gels were made in triplicate by using a minimum of three independent extractions. Conditions for isoelectric focusing and second dimension run are described in Supporting Information. 2-D gels were stained with silver nitrate as described by Shevchenko et al [18]. Preparative silver-stained gels were scanned with the Image scanner UMAX (Amersham Biosciences) and analyzed by ImageMaster 2D Platinum v.5 software (Amersham Biosciences). The determination of the relative spots volumes was performed by the software (mode: total spot volume normalization). For each analysis, statistical data (Student's t test) showed a high level of reproducibility between normalized spot volume of gels produced in triplicate. In particular statistical analysis of differential protein expression, evaluated in terms of \% volume intensity rate for each spot, was performed by the Student's T-test. Changes were considered significant at $P \leq 0.05(n=3$, standard deviation $\leq 0.01$ and $P \leq 0.05$ ). Relative results, evaluated in terms of spot intensity rate (Supporting Fig. 4S), have been obtained from a large number of spots (about 1000 for each reference maps) for which a systematic quantization was performed by comparing the different conditions described above. This enabled us to experimentally estimate the average error value associated with spot volume measurements from a very large number of determinations. For spots whose volume was significantly higher than background values ( 3 fold), the average error of measurements was approximately $20 \%$. Therefore, all relative variations outside the relative interval 0.66 (1/1.5) to 1.5 were considered to be significant, and submitted to Student's T test. In addition the 1000 differentially expressed spots obtained from image analysis were also screened on the basis of statistical significance criteria $(n=3$, standard deviation $\leq 0.01$ and $P \leq 0.05)$.

\subsection{Mass Spectrometry analysis}

Spot picking and digestion are described in Supporting Information. For data presentation we followed the Philadelphia Guidelines published on MCP website (http://mcponline.org/).

a) Protein identification by Peptide Mass Fingerprinting (PMF). In Peptide Mass Fingerprinting experiments the extracted peptides, desalted by reverse phase extraction using ZipTip C18 pipetting tips, were eluted with alpha-cyano-4hydroxycinnamic acid (HCCA) matrix $(0.05 \mathrm{mg} / \mathrm{ml})$ in acetonitrile/0.0.1\% TFA solution (1:1, [v/v]), and deposited on anchorchip target plate (Bruker-Daltonics, Bremen, Germany). MALDI-TOF data were obtained using an Reflex IV and Ultraflex III time-of-flight mass spectrometers (Bruker Daltonics) operating in reflectron positive mode and controlled by the FlexControl software package v2.0. Spectra were obtained by accumulating the signals of 50-200 laser shots. Details of spectra acquisition and processing are reported in Supporting Information. Briefly acquired spectra were processed by FlexAnalysis software v2.0 (BrukerDaltonics) filtered out the known peptides deriving from both human keratins and trypsin autoproteolysis. The created peak lists were analyzed by the MASCOT search engines v2.2.03 (http://matrixscience.com), Matrix Sciences by interrogating the NCBInr protein database v2008.10.10 (http://www. ncbi.nlm.nih.gov/), restricting the Taxonomy to Homo sapiens. The raw data acquired by Reflex IV and Ultraflex III time-offlight mass spectrometer (Bruker Daltonics) were converted into peaklist files for database searching. The query for database searching was performed with $\pm 100 \mathrm{ppm}$ as maximal tolerance, a single trypsin missed cleavage, carbamidomethyl of cysteine residues as fixed modification, and oxidation of methionine as variable modification. There were no restrictions regarding mass or PI for MASCOT based searches. Mascot protein scores greater than 66 were considered significant $(p<0.05)$ for protein identification assignment. The estimation of false positive rate was calculated according to Mascot default threshold (5\%). In some instances we have observed that tryptic peptides matched to multiple members of a 
protein family. We have performed in Peptide Mass Fingerprinting analysis the redundancy correction accepting the accession number corresponding to proteins confirmed by LC-MS/MS. In the case of chaperonin HSP60, (gil 306890; gi|49522865) we reported the accession numbers with highest score.

b) Data analysis and protein identification by LC-MS/MS. The digested peptides were also extracted from the gel in a solution containing $\mathrm{H}_{2} \mathrm{O}$ /acetonitrile (1:1, [v/v], 3 times) and pre-concentrated using a SpeedVac concentrator 5301. In nano-LC-MS/MS experiments the extracted peptides were analyzed on an ESI-Q-TOF mass spectrometer (Waters Corp., Manchester, UK) equipped with a CapLC system (Waters Corp., Milford, MA). Protein identification was based on MASCOT identification by mass homology, using MASCOT software v2.2 (http://matrixscience.com). Raw data files acquired by ESI-Q-TOF mass spectrometer (Waters Corp.) were automatically processed into peaklist files by using MassLynx server v4.0 (Waters Corp.) to obtain a Pkl format compatible for MASCOT MS/MS ion search engine. The estimation of false positive rate was calculated according to Mascot default threshold (5\%) to validate protein identification obtained by Peptide Mass Fingerprinting and by LC-MS/MS (Supporting Tables 1S and 2S). More detailed methods are described in Supporting Information. Software/methods to evaluate site assignment by MS was not used and the post-translational modification of a stathmin family member was only described as an evidence deriving from western blot analysis with ubiquitin antiserum.

c) Protein identification and quantitation by $\mathrm{LC}-\mathrm{MS}^{\mathrm{E}}$. In $\mathrm{LC}-\mathrm{MS} \mathrm{S}^{\mathrm{E}}$ experiments, ion detection, clustering, normalization, protein identifications and relative quantitation were obtained with the embedded ion accounting algorithm of ProteinLynx GlobalServer software v2.3 (Waters Corp.), as previously reported $[19,20]$. In solution protein digestion was performed as already described $[20,21]$. This novel approach for qualitative and relative quantitative analysis is based on experiments carried out without the use of isotopic labels, applying an ion accounting algorithm useful to performed an "Expression Analysis" [22,23]. How the PLGS Expression module works, applied data clustering and normalization and statistic analysis, has been previously described in detail by Silva JC, Huges MA et al [24-26]. Deeper description of data acquisition and processing used in this work are reported in Supporting Information. In the attempt to guarantee the biological reproducibility protein samples (different time point conditions) from pooled cellular extracts of different experiments $(n=3)$ were processed to tryptic digestion. Enolase from Saccharomyces cerevisiae protein digestion was used as internal standard, Peptide mixture for LC-MS were loaded on a nanoACQUITY UPLCTM System (Waters Corp.) coupled to a Q-Tof PremierTM mass spectrometer (Waters Corp.) operating in high-low mode. In the attempt to guarantee analytical reproducibility for each condition we performed three experiments in triplicate and we chose the most reproducible dataset for subsequent analysis. Protein identifications were obtained with the embedded ion accounting algorithm of the software and searching a UniProtKB/SwissProt database (http://www.ebi.ac.uk/swissprot),
(398.181 entries, indexed on 23 September 2008) restricted to Homo Sapiens taxonomy (25.245 entries), to which sequence of Enolase from Saccharomyces cerevisiae was appended. For database search the peptide (precursor ions) tolerance was set to $15 \mathrm{ppm}$ for MS while the fragments (product ions) tolerance was set to $20 \mathrm{ppm}$ in MS/MS. No criteria of redundancy correction after PLGS identifications were applied. Different isoforms/individual members of a protein family were reported since discriminated by PLGS software. However we reported in the Supporting Table 3S different protein isoforms having discriminating sequence peptides identified by LC$\mathrm{MS}^{\mathrm{E}}$ (at high collision energy). The false positive rate (FPR) of the identifications calculated by the algorithm (PLGS) is typically 3 to $4 \%$ with a randomized database, appended to the original one, which is five times the size of the original utilized database $[19,20]$. The interrogation of SwissProt database by ProteinLynx GlobalServer software (PLGS) (Waters Corp.) has provided a data table of the identified proteins and an EMRT table in which are listed the identified clusters (i.e., the detected accurate mass/retention time pairs identified). In addition for each replicate conditions of different cluster (Untreated, Epoxomicin 24 and $48 \mathrm{~h}$ ) we have calculated the percentage of Retention Time Coefficient of Variation (\%CV $\mathrm{RT} ; \leq 5 \%)$ the percentage of peptide Intensity Coefficient of Variation (\% CV I; $\leq 5 \%$, Gaussian distribution), the relative Standard Deviation on mass distribution (RSD; $\leq 12 \mathrm{ppm}$ ), (Fig. 2C, D and E). To evaluate triplicate reproducibility we have considered the logE normalized intensity EMRT scatter plots of two injections of the same sample (Fig. 2). For relative analysis, the identified proteins were normalized against P00924 entry (Enolase from Saccharomyces cerevisiae) (Supporting Tables 3S and 4S) while the most reproducible peptides for retention time and intensity deriving from protein digestion of Enolase were used to normalize the EMRT table. The lists of normalized proteins were screened according the following criteria: protein identified in at least 2 out of 3 injections of the same condition; proteins with $0<\mathrm{P}<0.05$ or $0.95<\mathrm{P}<1$ and proteins with a ratio of expression level within the conditions above 1.5 on decimal scale. See Supplemental Information for more details.

\subsection{Bioinformatic analysis}

a) Protein classification. PANTHER (Protein ANalysis THrough Evolutionary Relationships) Classification System (PANTHER Pathway®, version 2.5, released date 2009-01-06, www.pantherdb.org), was used for classification of proteins identified by $2 \mathrm{DE}$ and $\mathrm{LC}-\mathrm{MS}^{\mathrm{E}}$. This software allows to predict function using published scientific experimental evidence and evolutionary relationships. Proteins are classified by expert biologists into families and subfamilies of shared function, which are then categorized by molecular function and biological process ontology terms $[27,28]$.

b) Proteome wide pathway meta-analysis. Protein expression profiling, carried out on low-dose Epoxomicin responsive cells was analyzed using Ingenuity Pathways Analysis software (IPA; Ingenuity ${ }^{\circledR}$ Systems, version 8.6, released date 2010-05-28, www.ingenuity.com) a web-based application that enables the identification of relationships, biological mechanisms, functions, and pathways of relevance 
associated with the molecules under study. Information concerning genes, along with their fold-change values (0.6-1.5), were used as input to the Ingenuity Pathways Analysis program and algorithmically computed to convert their biological relationship into networks. In particular proteomics dataset was mapped to different focus genes (i.e., 29 for the first network characterized by score 66; Supporting Table 5S), used as starting point for generation of biological networks. The networks obtained derived from a process in which the application queries the Ingenuity Pathways Knowledge Base for physical and functional interactions among focus genes and all other genes (gene products) stored in the knowledge base. A relevancy score was generated using a p-value calculation indicating the likelihood that the assembly of a set of focus genes in a network could be explained by random chance alone. This score take into account the number of focus genes and the size of the network.

\subsection{Ubiquitin immunodetection and image analysis on SH-SY5Y cells}

SH-SY5Y cells were seeded at $30 \%$ confluence on glass coverslips and exposed to drugs as previously described. NB cells were fixed and permeabilized for $20 \mathrm{~min}$ in PBS containing 3\% paraformaldehyde , $0.1 \%$ glutaraldehyde , $0.1 \%$ Triton X-100, quenched with $50 \mathrm{mM}$ ammonium chloride and processed for labeling with monoclonal anti-ubiquitin $(0.4 \mu \mathrm{g} / \mathrm{ml}, 2 \mathrm{~h}, \mathrm{RT})$. Nuclei were counterstained with Hoechst dye $33258(1 \mu \mathrm{g} / \mathrm{ml})$. Secondary antibody coupled to Cy3-coniugated IgG antibody was used. Digital images were obtained with Leica TCS SP5 confocal microscope (Leica Microsystems, Wetzlar, Germany). Ubiquitin specific signal and Hoechst fluorescence were captured using sequential acquisition and adjusting both photomultiplier tube gain and offset to provide sub-saturating fluorescence intensity with optimal signal to noise ratio. Pictures were acquired using the LAS AF software v1.7.0 (Leica Microsystems) and analyzed using ImageJ v1.40 g software (www.rsb.info.nih.gov). Five randomly chosen Region of Interest (ROI) from different fields of the coverslips (corresponding to the same area of $150 \times 10^{3} \mu \mathrm{m}^{2}$ for each) were analyzed in at least three independent experiments. Pixel numbers in all images were counted to determine differences in the distribution of fluorescence between the different experimental groups, subtracting background fluorescence to normalize. Relative fluorescence data were exported from ImageJ generating histograms into KaleidaGraph v3.0 software for data presentation. Statistical analyses were performed using the paired t-test. Acquired images of separated channels were exported using Leica Application Suite 6000 as tiff files. Figures were generated using Image $\mathrm{v} 1.43 \mathrm{~d}$ software.

\subsection{Primary neuronal cultures}

Mixed spinal cord cultures were prepared from 15-day-old mouse embryos of a wild type female mated with a SOD1G93A male as described [29]. Briefly each neural tube was dissected and singularly incubated for $10 \mathrm{~min}$ in $0.025 \%$ trypsin and then dissociated by gentle trituration. The resulting mixed cultures were plated on BD BioCoat ${ }^{\mathrm{TM}}$ laminin and poly-D-lysine-coated glass cover slips (Sigma), (four cover slips for each spinal cord) and maintained in Neurobasal supplemented with 5\% FBS and 5\% HS. Two to three hours after plating the medium was replaced with Neurobasal supplemented with B-27, $0.5 \mathrm{mM}$ glutamine. Each embryo was screened for the presence of transgene SOD1G93A as described by Spalloni et al [29]. 12-14 DIV cultures were used for toxicity experiments. Immunocytochemistry on neuronal cultures was performed as NB cells. Confocal images were taken with LSM700 Zeiss.

\section{Results \\ 3.1. Evaluation of Epoxomicin biological effect and impact on NB proteome}

In order to investigate the pathways activated by protein accumulation in cells of neuronal origin, we used a model of neuroblastoma cell line treated with a proteasome inhibitor. In this study we have indeed propagated a NB SH-SY5Y population responsive to low-dose of Epoxomicin to examine the biological implications associated to UPS impairment. Treatment with different doses of Epoxomicin for $24 \mathrm{~h}$ results in inhibition of cell growth but no cell death even at the highest doses used (Fig. 1A). Treatment for longer time (up to $48 \mathrm{~h}$ ) results in cell death (Fig. 1B). This is due very likely to classical apoptosis as demonstrated by the increase of the sub-G1 peak of PI stained cells (Fig. 1C). Growth arrest is due to a block of the cells in the G2/M phase of the cell cycle (Fig. 1C). Interestingly we have observed that low-dose Epoxomicin exposure trigger to: i) a gradable and statistically significant increase in p53, p21 and caspase-8 levels (Supporting Fig. 1S); ii) the selection of an homogeneous, increased in dimension NB populations (Supporting Fig. 2S); iii and iv) a time-dependent accumulation of polyubiquitinated proteins and free-ubiquitin decreasing (Fig. 1) associated to the activation of macroautophagy (Supporting Fig. 3S).

Under this experimental conditions proteasome blockage indeed results in a time-dependent accumulation of polyubiquitinated proteins and reduction of free-ubiquitin that cause the increased dimension of NB populations (Fig. 1D). To investigate the changes in the proteome of NB cells following Epoxomicin treatment we used different proteomic approaches based on the combination of high-resolution 2-DE with MS (PMF and LC-MS/ MS). We compared untreated parental NB cells with cells exposed to Epoxomicin for 24 and $48 \mathrm{~h}$ by 2-DE. On each gel we have visualized an average of 1.000 spots. Total differentially spots (259 spots) were screened, as described in materials and methods, for fold change and statistic significance (19 spots) and filtered on the base of Mascot significant score (14 spots) getting to 14 distinct accession number IDs (Supporting Table 1S). Fig. 2A shows a representative gel, in which all the differentially expressed protein spots are squared and marked with numbers. The protein spots with the highest degree of modulation are shown at higher magnification in Fig. 2B. These protein spots have been divided in three groups based on their behavior upon Epoxomicin treatment: increased after 24 and 48 h; decreased after 24 and 48 h; transiently changed only after $24 \mathrm{~h}$ (Supporting Fig. 4S.A-C). All these spots have been further analyzed by MS, identified by Mascot database search and, excluding redundancy, were assigned to 14 distinct accession numbers IDs as reported in Supporting 

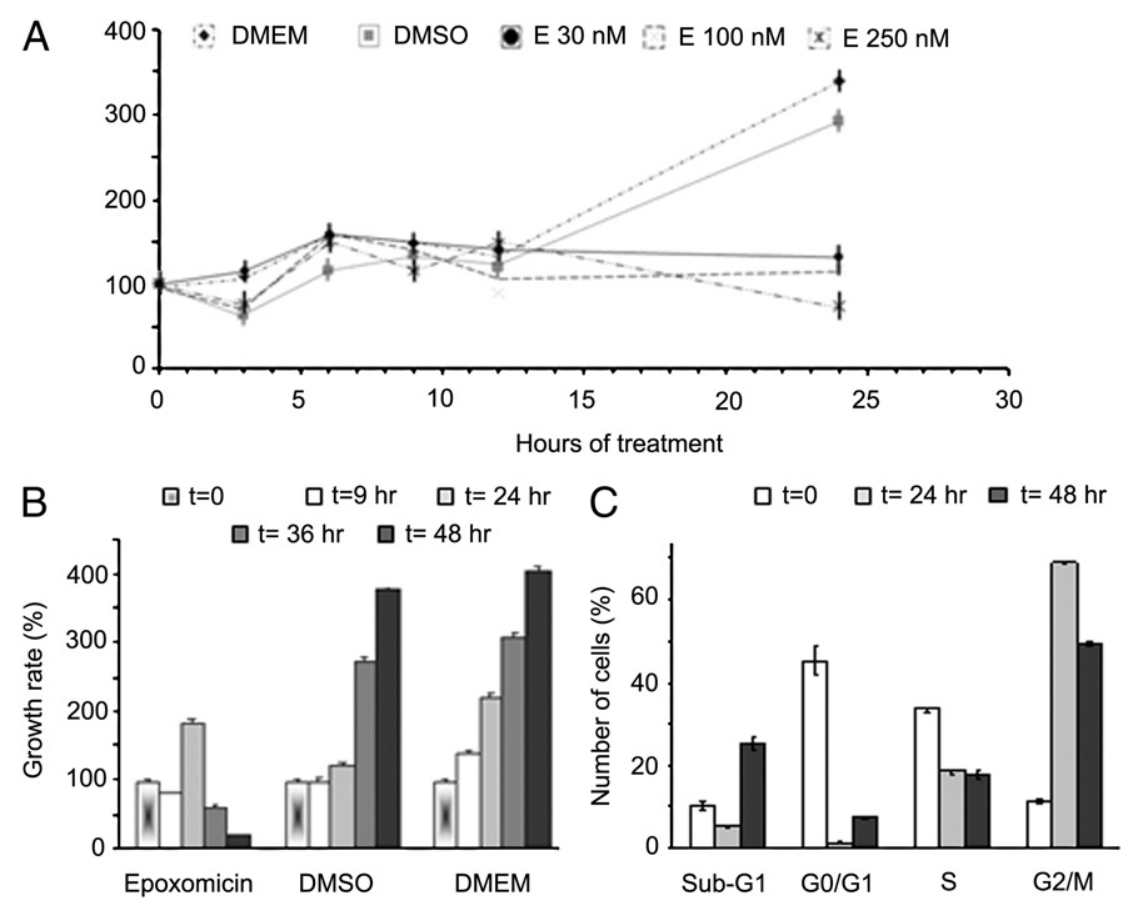

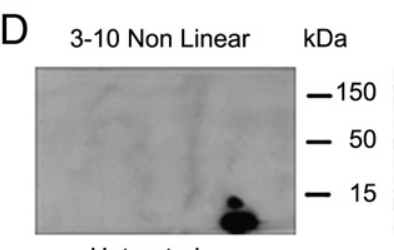

Untreated

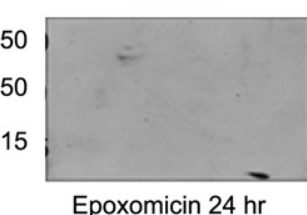

3-10 NL

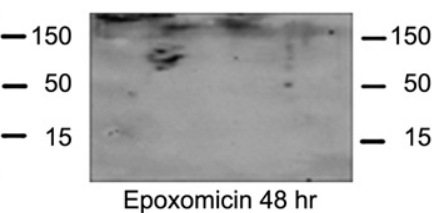

Fig. 1 - Evaluation of Epoxomicin blockage effects onto proliferation, cell death and ubiquitination in NB cells. Drug time and concentration dependent response assessment in NB SH-SY5Y cells. Low-dose of proteasome inhibitor ( $30 \mathrm{nM}$ ) has been chosen to examine the biological implications associated to UPS impairment (A). Data from vitality assays demonstrate that $30 \mathrm{nM}$ Epoxomicin exposure till $48 \mathrm{~h}$ trigger the proliferation of responsive cells (B) avoiding the strong induction of cellular apoptosis in few hours and supporting G2/M phase increase (C). Time-dependent accumulation of polyubiquitinated proteins and free-ubiquitin reduction is shown in 2-DE gels (D).

Table 1S. In addition we confirmed protein identifications by LCMS/MS data (Supporting Table 2S), including those regarding two proteins (5909 Moesin and 6565 Stathmin 1/oncoprotein 18) which have been initially identified with a low Mascot score (respectively 61 and 57) and a theoretical Isoelectric point (pI) and Molecular ratio (Mr) not in perfect agreement with those ensuing from experimental values.

Proteome changes of NB cells in response to Epoxomicin were also investigated by a complementary proteomic approach based on shotgun peptide profiling on nanoscale ultra performance LC-MS ${ }^{\mathrm{E}}$. This relative quantitation analysis utilizes the three most abundant peptides identified to a protein for normalization [30]. The relative ratios and standard deviations expressed in log scale are reported for differentially expressed proteins to demonstrate the accuracy of the relative quantitation measurements (Supporting Table 3S). This analysis allowed the identification of a higher number of differentially expressed proteins: 376 total proteins in untreated vs Epoxomicin $24 \mathrm{~h} ; 363$ in untreated vs Epoxomicin $48 \mathrm{~h}$ and 321 in Epoxomicin $24 \mathrm{~h}$ vs Epoxomicin $48 \mathrm{~h}$. Among them only 61 (58 up-regulated, 2 down-regulated and 1 protein with transient level changes) have been found significantly modified upon Epoxomicin treatment, while other proteins have been considered unique to each time point (41 in untreated condition; 11 in Epoxomicin $24 \mathrm{~h}$ and 27 in Epoxomicin $48 \mathrm{~h}$ condition) applying stringent filtering criteria. In addition to the stringent filtering criteria described in the experimental procedures we also filtered the 140 total proteins identified by Expression analysis reporting only those identified with at least 2 peptides with PLGS score $>30$ (116 distinct accession numbers IDs). The proteins identified by this approach could be divided in different functional groups including: stress induced proteins, structural proteins, enzymes and regulatory proteins, etc. (Supporting Tables $3 S$ and $4 S$ ). All the distinct protein identifications obtained by 2-DE electrophoresis and LC-MS ${ }^{\mathrm{E}}$ were examined for their known biological function and grouped in the respective functional category using Panther Classification System software (www. pantherdb.org), (Supporting Fig. 5S). Finally our proteomic results were validated by western blot analysis of a selected group of proteins (Supporting Fig. 6S) on different human NB cell lines (SH-SY5Y, SK-NAS, LAN-5 and IMR-32) characterized by distinct genetic background. In particular we have chosen to confirm the differential expression of some identified 


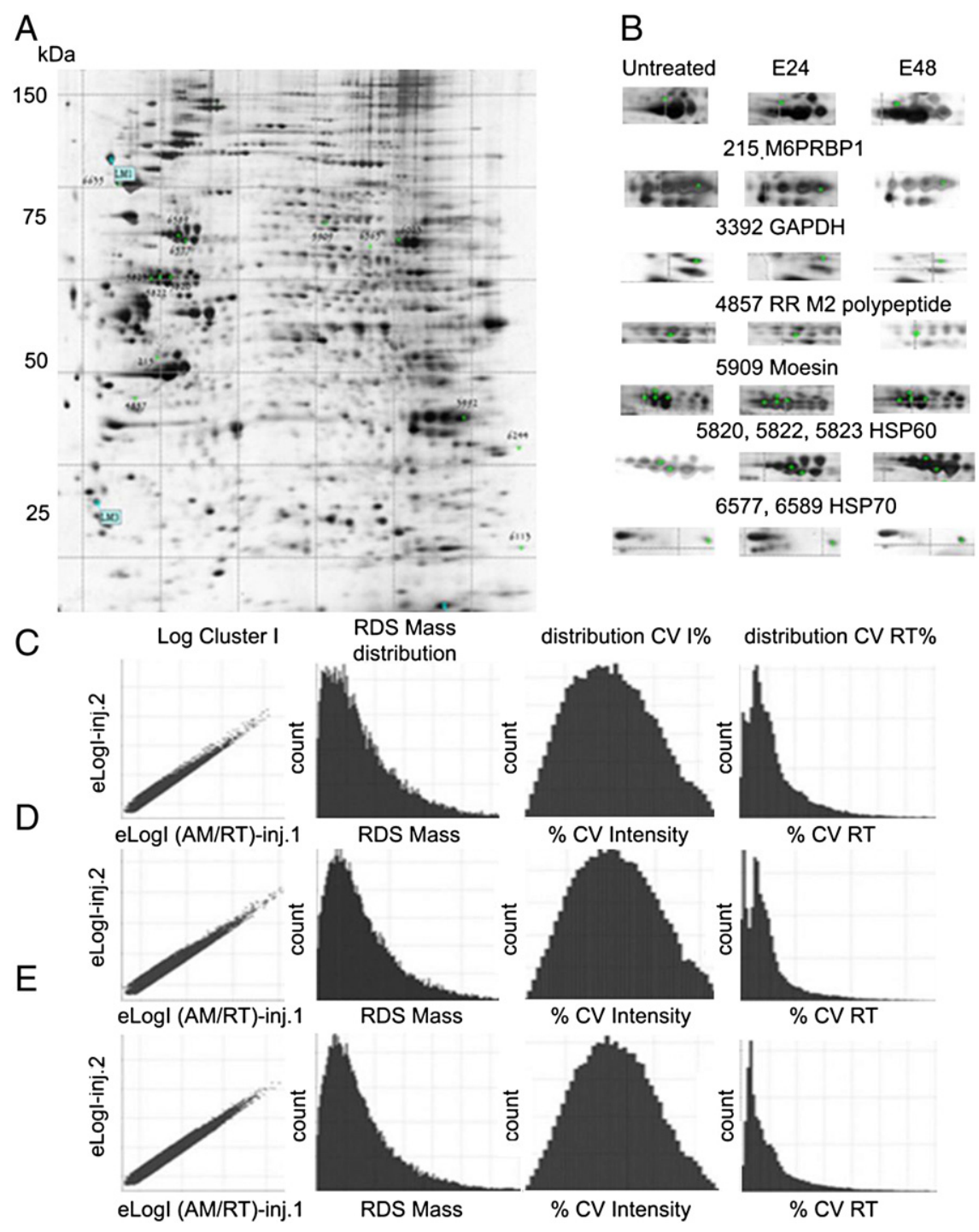

Fig. 2 - Epoxomicin impact on NB proteome assessment by 2-DE coupled with MS and shotgun proteomics experiments. 2-DE reference gel with all the differentially expressed proteins marked and enclosed in boundaries (A). Snapshot of the most statistically significant differentially expressed protein spots (B). Panel C-E show data quality evaluation of data-set parameters obtained from the "Expression analysis" of NB untreated cells (C), NB cells exposed to Epoxomicin for $24 \mathrm{~h}$ (D) and $48 \mathrm{~h}$ (E). i) In Log Cluster I column the Binary comparison of the log intensity measurements obtained from the matched EMRT cluster for two replicate injections for every condition is shown. The 45-degree diagonal line indicates the absence of variation through the detected range; ii) The RSD (Relative Standard Deviation) column shows the relative standard deviation barchart from all the EMRT components within \pm 5 ppm of the mean mass measurements. iii) The CV I\% (Coefficient of Variation) column shows the average coefficient of variation of the signal intensity of the clusters. iv) The CV RT\% (Retention Time) column shows the average retention time coefficient of variation centered at $0.4 \%$.

proteins, TIP-47 for its role in inclusions drain [31] and HSPs for their involvement in CMA for the executing of aggresome clearance [32]. In addition we have performed an immunoblot analysis to confirm the identification of two proteins (VGF nerve growth factor inducible precursor and Stathmin 1/oncoprotein 18) whose theoretical pI or Mr was not in perfect agreement with the experimental values. Interestingly, by western blot analysis, we have observed a putative post-translational modified form of stathmin family members (Supporting Fig. 7S.A, B) and confirmed the decrease of relative amounts of VGF nerve growth factor inducible precursor in response to Epoxomicin exposure (Epoxomicin 48 h), (Supporting Fig. 7S.C).

\subsection{Proteome-wide pathway meta-analysis}

All the NB expressed proteins modulated by Epoxomicin lowdose treatment resulted by different comparative proteomics approaches were classified for their molecular functions by PANTHER Classification System (Fig. 5S). Most part of the differential expressed proteins were represented by Nucleic acid binding proteins $(21.5 \%)$, Chaperones proteins (14.6\%), Proteins with miscellaneous functions (10.3\%) and Cytoskeletal proteins (13.9\%). Select regulatory proteins (5.6\%), Proteins with different other functions (5.5\%), Select calcium binding proteins (4.2\%), Oxidoreductases (3.5\%), Hydrolase, Isomerases and Transfer/ 
carrier proteins (2.8\%), Signaling molecules and Transcription factors $(2.1 \%)$, Transporters (1.4\%), Lyase (1.3\%), ion channels and different enzymes $(0.7 \%)$ were also well represented. To highlight possible molecular mechanisms underlying protein repertoire impact in response to UPS blockage, a pathway meta-analysis was carried out on the complete proteomics datasets of modulated identified proteins by using Ingenuity Pathway Analysis software (IPA), (Fig. 3). IPA provides an unsupervised insight through complex biological networks responding to injuring drug stimuli that enables the discovery of relevant networks contributing to initial experimental paradigms. All the 123 identified proteins, along with their foldchange expression values, were converted in the relative genes and uploaded into IPA program generating a genomics dataset that mapped to several genes in the IPKB. Physical and functional interactions between focus gene and all the genes, stored in the knowledge base, have been used to build networks. In particular IPKB provided eight networks, calculating a significance score for each network, and revealing novel interactions. In addition for each network, gene involvement in pathophysiological conditions (i.e., Neurological Disease, Genetic Disorders, Metabolic Diseases, etc.) and the executed functions (i.e., Survival and Cytotoxicity, Cellular Function and Maintenance, Cellular Assembly and Organization, etc.) were elucidated (Supporting Table 5S). By merging the elicited networks we have highlighted a more complex pathway converging on betaestradiol (Fig. 3). Since the neuroprotective role of beta-estradiol is well documented [33] we further investigated its role in our model.

\subsection{Effect of beta-estradiol treatment on proteasome blockage by Epoxomicin}

To evaluate the effect of estradiol [34-36] on low-dose Epoxomicin treatment of NB cells we treated SH-SY5Y cells with betaestradiol for $24 \mathrm{~h}$ before exposing them to Epoxomicin for 24 and $48 \mathrm{~h}$. Our results show that while estradiol treatment has a modest effect on cell cycle distribution of Epoxomicin treated cells for both 24 and $48 \mathrm{~h}$ (Fig. 4A). It significantly protects them from apoptosis as shown by histogram relative to sub-G1 cells (Fig. 4B). The effect on cell survival is paralleled by an effect on the accumulation of polyubiquitinated proteins (Fig. 5A, B). In addition immunofluorescence experiments using antibodies against ubiquitin show that beta-estradiol treatment results in a decreased number of cells containing ubiquitin aggregates (Fig. 5B). Moreover these aggregates are no longer localized around the nucleus but are spread in the cytoplasm and are decreased in intensity, calculated using the Image J software, as described in Materials and methods section.

Altogether these data show that treatment with estradiol results in increased ability of cells to deal with accumulated proteins that cannot be eliminated through the proteasome by activating a distinct degradation pathway, this in turn results in increased cell survival. One possibility to explain how $\beta$-estradiol treatment results in removal of protein aggregates thus protecting cells from death is the activation of the autophagic pathway. We therefore investigated this possibility. As shown in Fig. 6A staining of SH-SY5Y with lysotracker and an anti ubiquitin antibody shows that pre-treatment with $\beta$-estradiol results in increased autophagolysosome formation paralleled by reduced ubiquitinated protein aggregates. Western blot for LC3 confirms autophagosome formation, indeed Fig. 6B-C show conversion of LC3-I in LC-II, the form linked to autophagy activation. Interestingly treatment with $\beta$-estradiol alone does not induce autophagy but only in combination with proteasome blockage.

\section{4. $\boldsymbol{\beta}$-Estradiol and Epoxomicin impact on primary spinal cord cultures}

In the attempt to confirm the data obtained in our NB model in this study we have used primary neuronal cells, specifically mixed spinal cord cultures. Neurons were treated with $\beta$ estradiol $(24 \mathrm{~h})$ before the exposure to Epoxomicin $(24 \mathrm{~h})$, then the presence of polyubiquitinated proteins aggregates was evaluated. As shown in Fig. 7 ubiquitin positive aggregates (green) increased in the neurons (MAP2, red) following Epoxomicin treatment, compared to non treated neurons (Untreated), (Fig. 7 A-F). $\beta$-estradiol pre-treatment $(24 \mathrm{~h}$ ) reduced ubiquitin staining intensity and the number of ubiquitin positive aggregates (Fig. 7C, F). The presence of polyubiquitinated aggregates is an hallmark of neurodegenerative diseases $[37,38]$ including Alzheimer's Disease, Parkinson's Disease and Amyotrophic Lateral Sclerosis (ALS). Thus we have investigated Epoxomicin's effects on an in vitro model of ALS. Mixed spinal cord cultures over-expressing the ALS-affecting mutation G93A on the superoxide dismutase 1 gene (SOD1), challenged with Epoxomicin $(24 \mathrm{~h})$ show an increase in ubiquitin intensity compared to non treated cultures (Untreated), (Fig. 7 E vs D). Again, $\beta$-estradiol pre-treatment $(24 \mathrm{~h})$ was able to decrease ubiquitin positivity (Fig. 7F vs E).

\section{Discussion}

Although intracellular protein accumulation and aggregation are characteristics of many different neurological disorders the relationship between protein aggregates accumulation and pathogenesis has not been defined yet [39-44]. Indeed protein inclusions have been suggested to be toxic, protective or simply correlate with the disease without influencing its pathogenesis. Despite this, many reports show that removal of protein aggregates has a protective role on neurons and can lead to symptom regression [39,45-47]. Interestingly a common feature of accumulated proteins in proteinopathies is that they are ubiquitinated [48-50], raising the question of why they are then not eliminated through the proteasome. It is therefore of uttermost importance to identify pathways activated by protein accumulation and potential ways to induce their removal. Proteasome inhibition in vitro and in vivo has been often used as a model to study the link between protein accumulation and NDs onset [7-9]. In particular PIs, have been used to study NDs in which the main feature is peri-nuclear inclusion formations.

Here we analyzed changes in proteome following proteasome inhibition using an in vitro model. In our system neuroblastoma cells were treated with low doses of Epoxomicin, a well known proteasome inhibitor. Under these experimental conditions neuroblastoma cells show morphological changes characteristic of neurodegenerative diseases including formation of ubiquitinated inclusion agglomerates. Moreover low 


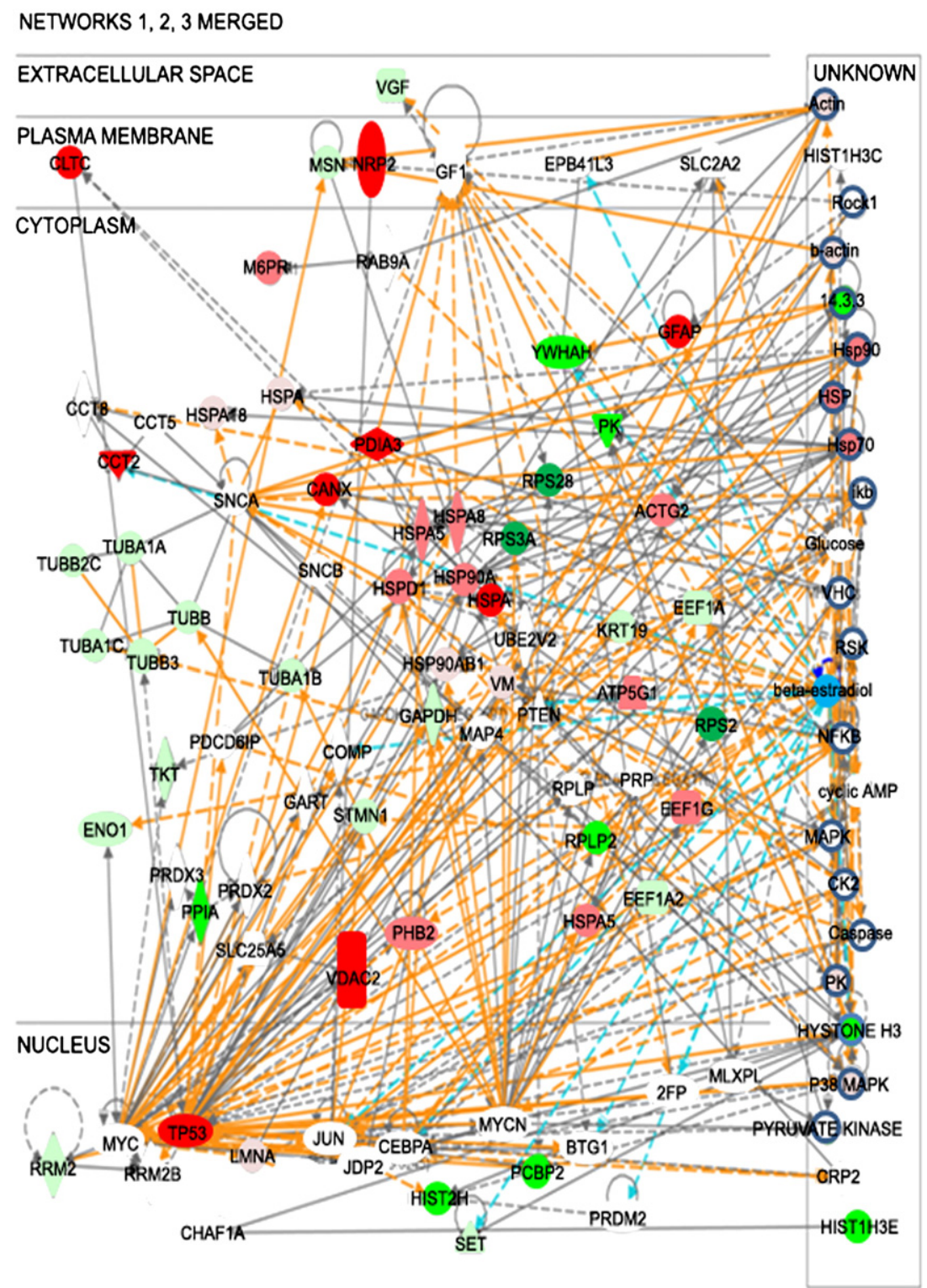

Fig. 3 - Ingenuity Pathway Analysis of Epoxomicin responsive cells. Proteomics dataset were converted in the relative genome dataset and used to generate a complex merging biological network. The intensity of the node color indicates the degree of up-(red) or down-(green) regulation: the higher the fold increase, the more intense red nuance. The analysis of merging networks shows the linkage among the modulated experimental dataset and beta-estradiol, the "core" of the resulting top merged networks. Orange lines highlight the beta-estradiol (bordered and displayed in the center of the figure) direct and indirect connections (orange lines). In this diagram molecules that are part of the same canonical pathway are indicated with a blue line. All the identified networks (8) are correlated with different Functions (i.e., Cell Death, Survival and Cytotoxicity, etc.), Relevant Diseases (i.e., Neurological Disease, Genetic Disorders, Metabolic Diseases, etc.) and overlay with a series of Canonical Pathways, such as Amyotrophic Lateral Sclerosis signaling, ERK/MAPK signaling, etc.

dose treatment results in only a modest effect on cell cycle and apoptosis, at least at early time points, allowing further biochemical analysis.

MS analysis of the proteome before and after Epoxomicin treatment has allowed the identification of a number of differentially expressed proteins involved in different cellular functions. We found an altered expression of enzymes essential for brain metabolic activity (i.e., Glyceraldehyde-3-Phosphate Dehydrogenase, Transketolase); reduction of important growth factors (VGF Nerve Growth Factor Inducible Precursor); transient induction of stathmin family members, known as microtubule destabilizers, possibly responsible for cytoskeleton alterations. In our study we obtained different results by the two functional proteomic approaches used (the 2-Dimensional Electrophoresis separation combined with Mass Spectrometry identification and the Shotgun Proteomics) by both a quantitatively (14 in 2D, 61 with LC-MS) and qualitatively (79 unique proteins for all the condition; untreated, Epoxomicin $24 \mathrm{~h}$ and 


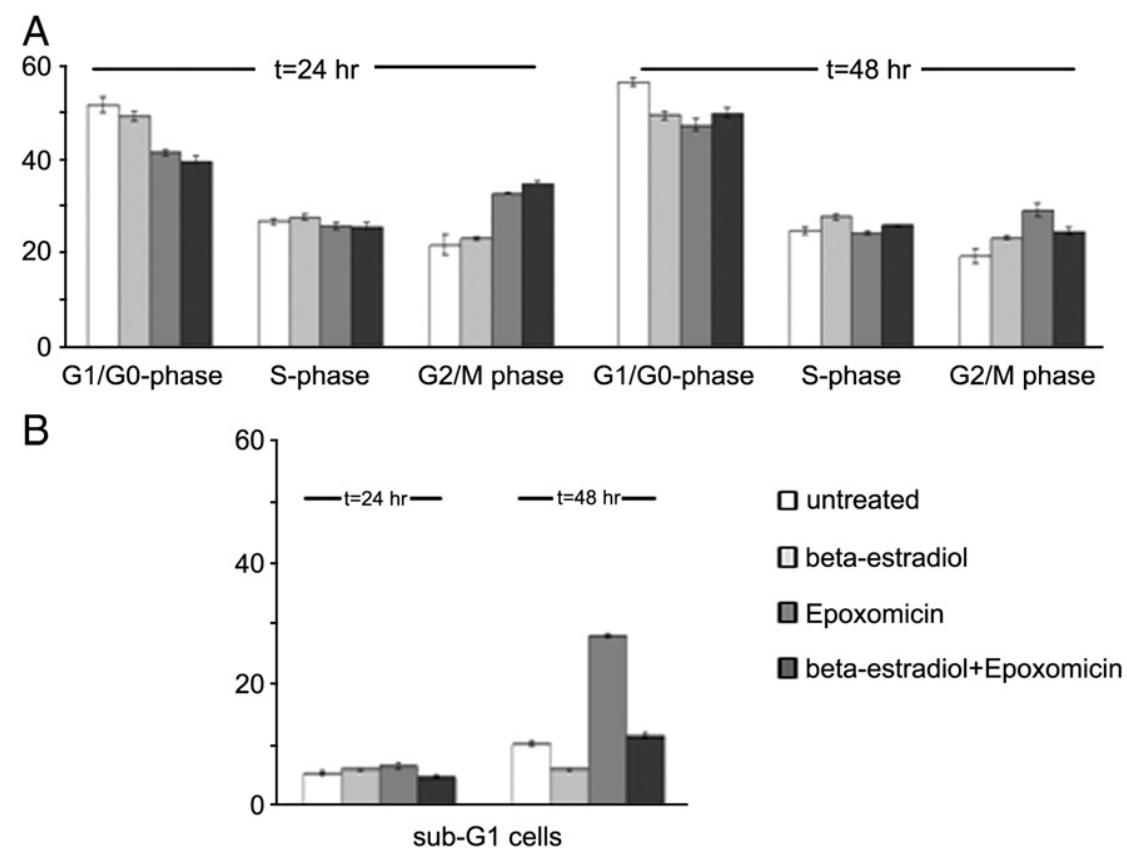

Fig. 4 - Evaluation of beta-estradiol pre-treatment effect in Epoxomicin NB treated cells. Cell cycle distribution and apoptosis induction measurements of NB cells exposed to beta-estradiol and Epoxomicin disclose an apoptotic bodies reduction (B) and an increase of cells rate in S-phase (A). Histogram shown have been drawn by using Flow cytometric analysis data in which cellular DNA content in apoptosis or necrosis and cell cycle distribution are performed by using both AV and 7-AAD (A) and PI dyes (B).

A

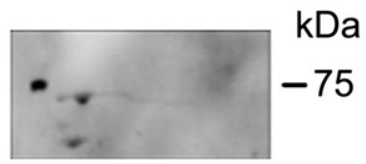

E24

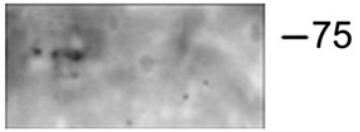

b-e+E24

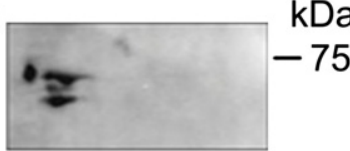

E48

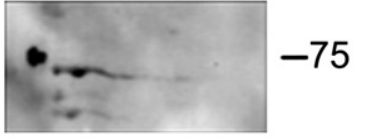

$b-e+E 48$

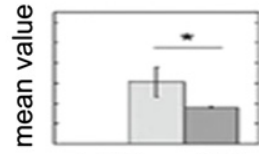

$\mathrm{E} 24 \mathrm{~b}-\mathrm{e}+\mathrm{E} 24$

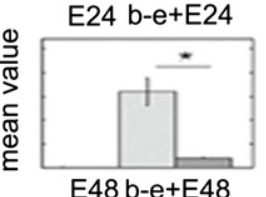

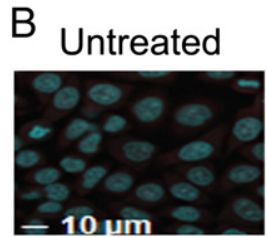
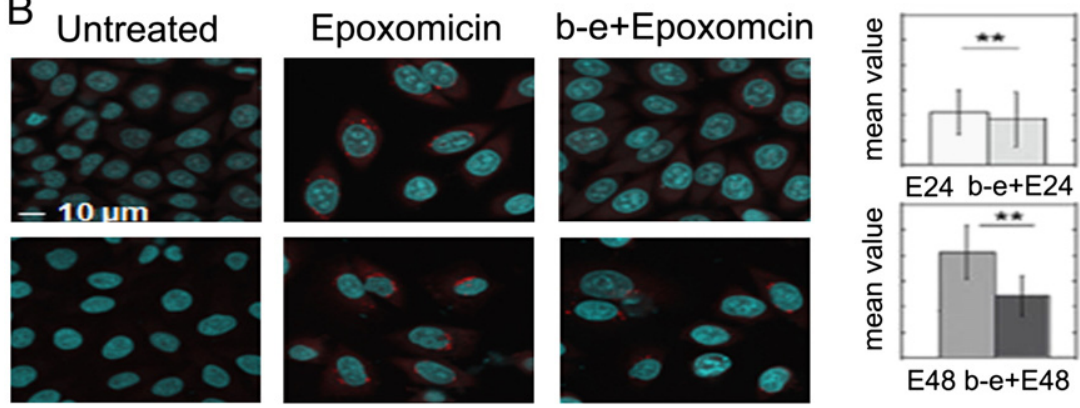

DAPI

ubiquitin

Fig. 5 - beta-Estradiol effects on the accumulation of polyubiquitinated inclusions in low-dose Epoxomicin responsive NB cells. (A) Immunoblots with ubiquitin antisera on 2-DE gels of NB cellular sample exposed to Epoxomicin or beta-estradiol followed by Epoxomicin. In this panel is clearly shown that in samples pretreated with beta-estradiol there is a strong reduction of ubiquitinated inclusions. The strong decrease of ubiquitin aggregates in cells treated with beta-estradiol and Epoxomicin is confirmed by ubiquitin immunostaining experiments were nuclei are stained in Blu with DAPI and Ubiquitin is stained in Red (B). The statistically significant reduction of aggregates in dimension and intensity, calculated as described in Materials and methods represented in the histograms. 
A

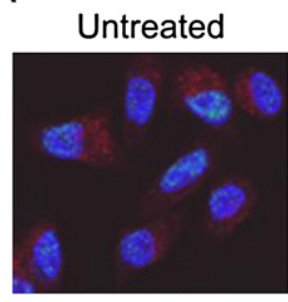

beta-estradiol

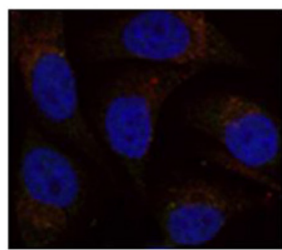

B b-e+
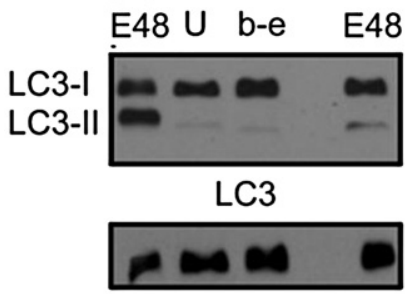

beta-actin

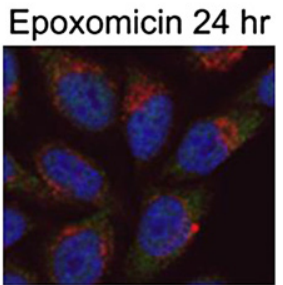

Epoxomicin $48 \mathrm{hr}$

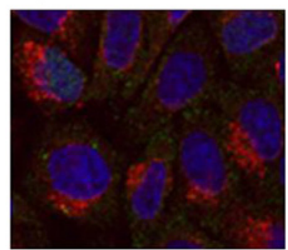

C beta-estradiol+

Epoxomicin $24 \mathrm{hr}$

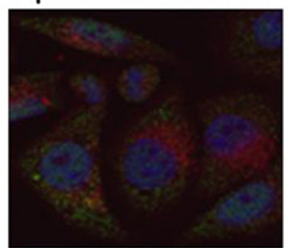

beta-estradiol+

Epoxomicin $48 \mathrm{hr}$

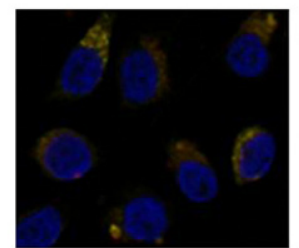

LC3-I L LC3-II

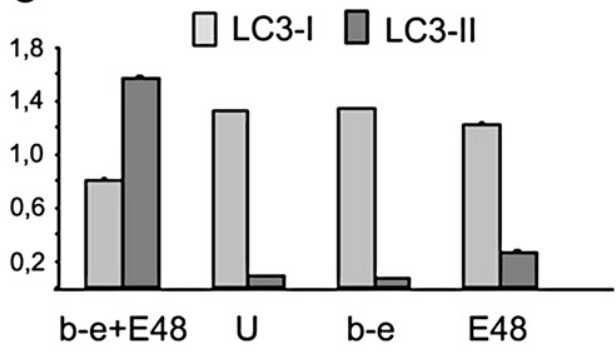

ubiquitin $\quad \square$ lysotracker

DAPI

Fig. 6 - Investigation on autophagic pathway induction in NB cells exposed to both beta-estradiol (24 h) and Epoxomicin ( 24 and $48 \mathrm{~h}$ ). Ubiquitin (green) and Lysotracker (red) immunodetection experiments demonstrate the autophagolysosome vacuole induction (yellow) in our cell model responsiveness to both beta-estradiol and Epoxomicin (A). Panels B (lane 1) show the increase of the autophagosome-associating form (LC3-II) and LC3-I decrease in samples of NB cells pretreated with beta-estradiol and exposed to proteasome inhibitor. The microtubule-associated protein 1 light chain 3 (LC3), immediately following the synthesis is cleaved to LC3-I form (18 kDa). During an autophagic process, a subpopulation of LC3-I is further converted to LC3-II form $(16 \mathrm{kDa})$. In this panel is shown the statistically significant decrease of LC3-I and increase in the expression of LC3-II as marker of autophagy induction. Density of specific bands was measured by using Image J. Values are means $\pm \mathrm{SE}$. ${ }^{*} \mathrm{P}<0.05,{ }^{* *} \mathrm{P}<0.001$, ${ }^{* * *} \mathrm{P}<0.0001(\mathrm{C})$.

Epoxomicin $48 \mathrm{~h}$ conditions) point of view. 2D electrophoresis represents the most employed technique for separating and quantifying intact proteins. In the case of 2-DE analysis differential expression among spots belonging to the same proteins may be highlighted, either when spots correspond to different aminoacidic sequences, or when post translational modifications cause a shift position on the gels. In the case of nLC-MSE although it may be possible to identify different isoforms with different aminoacidic sequences it was not always possible to discriminate among different isoforms (with the same aminoacidic sequences) due to post-translational modifications. The combination of the two approaches may be a useful strategy in order to obtain a more complete picture of the investigated protein repertories avoiding technique redundancy. Moreover, the partial overlapping of these two different experiments have been already reported in different systems and papers [20,21].

Altogether proteomics data have been suggestive of a group of major alterations that will eventually lead to cell death. Interestingly the meta-analysis of our data indicated a complex molecular pathway converging on beta-estradiol, prompting us to further investigate the role of this molecule in protein accumulation.

Although the neuroprotective properties of beta-estradiol [51] have been already reported in different diseases and models [52,53], and estrogen effects on neuronal viability have been demonstrated both in vitro and in in vivo [54,55], the underlying molecular mechanisms are still elusive. Our data indicate that beta-estradiol treatment is neuroprotective since it results in increased cell survival and proliferation, accompanied by reduction of ubiquitinated protein inclusions. These data were also confirmed on primary neuronal cultures. Moreover our data suggest that the effect on cell growth and viability is a consequence of protein clearance through activation of the autophagic pathway. Interestingly betaestradiol treatment only activates macroautophagy in cells in which a protein overload was induced through proteasome inhibition and not in untreated cells. Consistent with a role of 

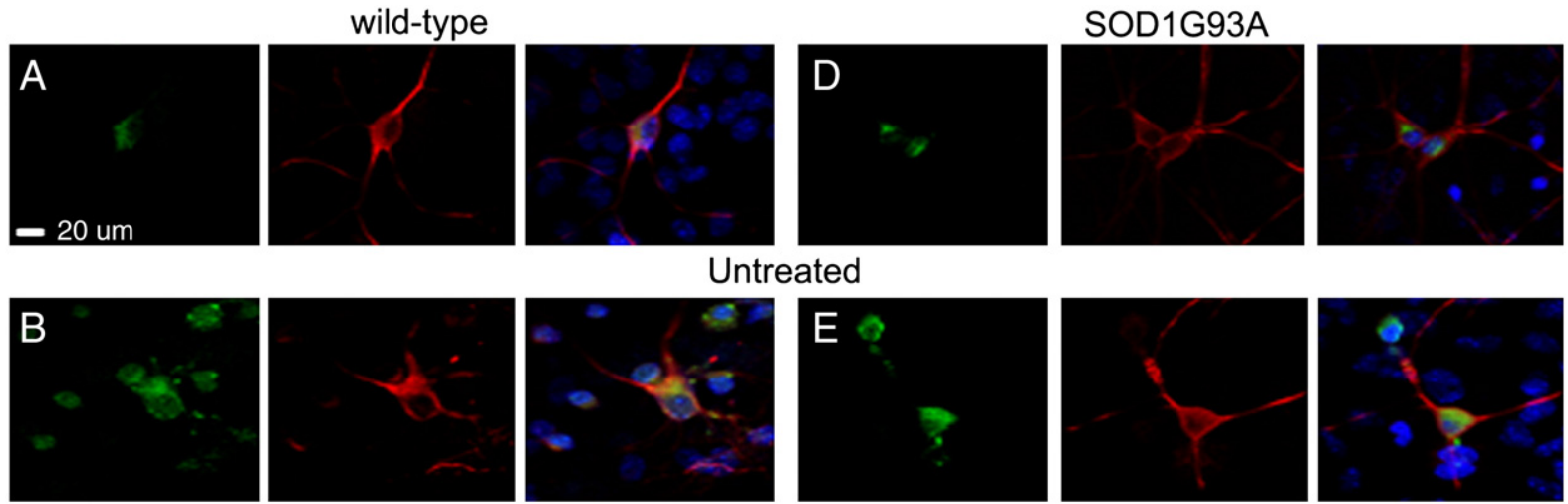

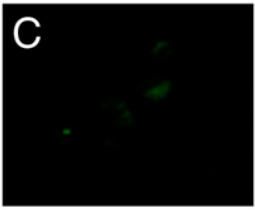

DAPI

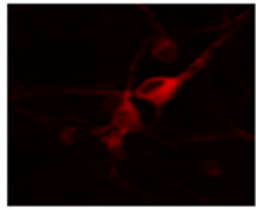

MAP-2

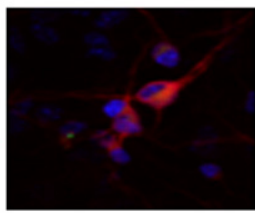

beta-estradiol+ Epoxomicin $24 \mathrm{hr}$
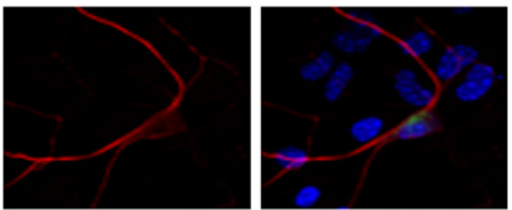

Fig. 7 - Evaluation of $\beta$-estradiol effects on the accumulation of polyubiquinated proteins in low-dose Epoxomicin responsive wild type and SOD1G93A mixed spinal cord cultures. Ubiquitin immunofluorescence (green) shows a decrease of ubiquitin aggregates in neuronal cells (MAP2, red) treated with $\beta$-estradiol for $24 \mathrm{~h}$ before the Epoxomicin challenge in both wild type and SOD1G93A cultures (respectively C and F) when compared to Epoxomicin alone (respectively B and E) and in untreated cells (respectively A and D). In blue are stained the nuclei with Hoechst.

autophagy as a protective mechanism upon protein accumulation is the finding that several proteins, known for their important role in the disposing of molecules to lysosomes for degradation, showed differential expression $[56,57]$. These include: TIP-47, HSP60 and 70. TIP-47 has been described as a cargo adaptor protein involved in endosomal MPRs collection into transport vesicles for Golgi complex [56]. HSPs have been generally described as chaperones, protein transporters/classifiers and cellular stress markers [52]. Interestingly in conditions of UPS impairment, HSP70 kDa has been shown to participate in the CMA to execute clearance of aggresomes [57].

\section{Conclusions}

In conclusion we have performed a comparative proteomic analysis on a human NB neuroblastoma cell line responsiveness to low-dose of Epoxomicin to estimate the rebound of UPS blockage on and protein functional networks involved in UPS impairment. As expected UPS inhibition by Epoxomicin orchestrates different cellular processes, whose deregulation may alter cellular homeostasis and led to apoptosis and accumulation of ubiquitinated peri-nuclear inclusions. In particular we have observed the modulation of expression of different proteins involved in inclusions drain and in CMA for the executing of inclusions clearance. Moreover we found experimental evidences of possible involvement of beta-estradiol in the aggresome removal by autophagy induction. We indeed believe that altogether these data support the idea that autophagy can play an important protective role in proteinopathies and strongly suggest a potential therapeutic use of beta-estradiol in neurodegenerative diseases as a mean to induce autophagy and remove accumulated proteins.

\section{Acknowledgment}

We thank Roberta Possenti and Andrea Levi for suggestions and for providing selective anti-VGF antibodies, Liliana Minichiello for critical reading of the manuscript and Fulvio Florenzano for several suggestions in the experimental procedures. This work has been supported by the "Rete Nazionale di Proteomica", FIRB RBRN07BMCT Project. Potential conflict of interest: Nothing to report.

\section{Appendix A. Supplementary data}

Supplementary data to this article can be found online at doi:10. 1016/j.jprot.2011.11.014.

\section{R E F E R E N C E S}

[1] Jacinto E, Hall MN. Tor signalling in bugs, brain and brawn. Nat Rev Mol Cell Biol 2003;4:117-26.

[2] Rubinsztein DC. The roles of intracellular proteindegradation pathways in neurodegeneration. Nature 2006;443:780-6. 
[3] Cuervo AM. Autophagy: in sickness and in health. Trends Cell Biol 2004;14:70-7.

[4] Nedelsky NB, Todd PK, Taylor JP. Autophagy and the ubiquitin-proteasome system: collaborators in neuroprotection. Biochim Biophys Acta 2008;1782:691-9.

[5] Pan T, Kondo S, Le W, Jankovic J. The role of autophagy-lysosome pathway in neurodegeneration associated with Parkinson's disease. Brain 2008;131:1969-78.

[6] Ciechanover A. The ubiquitin-proteasome pathway: on protein death and cell life. EMBO J 1998;17:7151-60.

[7] McNaught KS, Olanow CW. Proteasome inhibitor-induced model of Parkinson's disease. Ann Neurol 2006;60:243-7.

[8] Rockwell P, Yuan H, Magnusson R, Figueiredo-Pereira ME. Proteasome inhibition in neuronal cells induces a proinflammatory response manifested by upregulation of cyclooxygenase-2, its accumulation as ubiquitin conjugates, and production of the prostaglandin PGE(2). Arch Biochem Biophys 2000;374:325-33.

[9] D’Alessandro A, Pieroni L, Ronci M, D’Aguanno S, Federici G, Urbani A. Proteasome inhibitors therapeutic strategies for cancer. Recent Pat Anticancer Drug Discov 2009;4:73-82.

[10] McNaught KS, Perl DP, Brownell AL, Olanow CW. Systemic exposure to proteasome inhibitors causes a progressive model of Parkinson's disease. Ann Neurol 2004;56:149-62.

[11] McNaught KS, Björklund LM, Belizaire R, Isacson O, Jenner P, Olanow CW. Proteasome inhibition causes nigral degeneration with inclusion bodies in rats. Neuroreport 2002;13:1437-41.

[12] Venezia V, Russo C, Repetto E, Nizzari M, Violani E, Carlo P, et al. Apoptotic cell death and amyloid precursor protein signaling in neuroblastoma SH-SY5Y cells. Ann N Y Acad Sci 2004;1030:339-47.

[13] Waters WR, Harkins KR, Wannemuehler MJ. Five-color flow cytometric analysis of swine lymphocytes for detection of proliferation, apoptosis, viability, and phenotype. Cytometry 2002;48:146-52.

[14] Kalejta RF, Shenk T, Beavis AJ. Use of a membrane-localized green fluorescent protein allows simultaneous identification of transfected cells and cell cycle analysis by flow cytometry. Cytometry 1997;29:286-91.

[15] Cheung CK, Mak YT, Swaminathan R. Automated trichloroacetic acid precipitation method for urine total protein. Ann Clin Biochem 1987;24:140-4.

[16] Görg A, Obermaier C, Boguth G, Harder A, Scheibe B, Wildgruber $\mathrm{R}$, et al. The current state of two-dimensional electrophoresis with immobilized pH gradients. Electrophoresis 2000;21:1037-53.

[17] O'Farrell PZ, Goodman HM, O'Farrell PH. High resolution two-dimensional electrophoresis of basic as well as acidic proteins. Cell 1977;12:1133-41.

[18] Shevchenko A, Wilm M, Vorm O, Mann M. Mass spectrometric sequencing of proteins silver-stained polyacrylamide gels. Anal Chem 1996;68:850-8.

[19] Vissers JP, Langridge JI, Aerts JM. Analysis and quantification of diagnostic serum markers and protein signatures for Gaucher disease. Mol Cell Proteomics 2007;6:755-66.

[20] D’Aguanno S, D'Alessandro A, Pieroni L, Roveri A, Zaccarin M, Marzano V, et al. New insights into neuroblastoma cisplatin resistance: a comparative proteomic and meta-mining investigation. J Proteome Res 2011;4(10):416-28.

[21] De Canio M, D'Aguanno S, Sacchetti C, Petrucci F, Cavagni G, Nuccetelli M, et al. Novel IgE recognized components of lolium perenne pollen extract: comparative proteomics evaluation of allergic patients sensitization profiles. J Proteome Res 2009;8:4383-91.

[22] Li GZ, Vissers JP, Silva JC, Golick D, Gorenstein MV, Geromanos SJ. Database searching and accounting of multiplexed precursor and product ion spectra from the data independent analysis of simple and complex peptide mixtures. Proteomics 2009;9:1696-719.
[23] Geromanos SJ, Vissers JP, Silva JC, Dorschel CA, Li GZ, Gorenstein MV, et al. The detection, correlation, and comparison of peptide precursor and product ions from data independent LC-MS with data dependant LC-MS/MS. Proteomics 2009;9:1683-95.

[24] Silva JC, Denny R, Dorschel CA, Gorenstein M, Kass IJ, Li G-Z, et al. Quantitative proteomic analysis by accurate mass retention time pairs. Anal Chem 2005;77:2187-200.

[25] Silva JC, Denny R, Dorschel CA, Gorenstein MV, Li GZ, Richardson K, et al. J. Mol Cell Simultaneous qualitative and quantitative analysis of the Escherichia coli proteome: a sweet tale. Proteomics 2006;5:589-607.

[26] Hughes MA, Silva JC, Geromanos SJ, Townsed CA. Quantitative Downloaded from www.mcponline.org by on September 2, 2009, 35 proteomic analysis of drug-induced changes in mycobacteria. J Proteome Res 2006;5:54-63.

[27] Thomas PD, Campbell MJ, Kejariwal A, Mi H, Karlak B, Daverman R, et al. PANTHER: a library of protein families and subfamilies indexed by function. Genome Res 2003;13: 2129-41.

[28] Mi H, Dong Q, Muruganujan A, Gaudet P, Lewis S, Thomas PD. PANTHER version 7: improved phylogenetic trees, orthologs and collaboration with the Gene Ontology Consortium. Nucleic Acids Res 2010;38:D204-10.

[29] Spalloni A, Albo F, Ferrari F, Mercuri N, Bernardi G, Zona C, et al. Cu/Zn-superoxide dismutase (GLY93-> ALA) mutation alters AMPA receptor subunit expression and function and potentiates kainate-mediated toxicity in motorneurons in culture. Neurobiol Dis 2004;15:340-50.

[30] Silva JC, Gorenstein MV, Li G-Z, Vissers JPC, Geromanos SJ. Absolute quantification of proteins by LCMSE. A virtue of parallel MS acquisition. Mol Cell Proteomics 2006;5: 144-56.

[31] Díaz E, Pfeffer SR. TIP47: a cargo selection device for mannose 6-phosphate receptor trafficking. Cell 1998;93:433-43.

[32] Kaushik S, Cuervo AM. Chaperone-mediated autophagy. Methods Mol Biol 2008;445:227-44.

[33] Morissette M, Al Sweidi S, Callier S, Di Paolo T. Estrogen and SERM neuroprotection in animal models of Parkinson's disease. Mol Cell Endocrinol 2008;290:60-9.

[34] Reed JL, Dimayuga FO, Davies LM, Keller JN, Bruce-Keller AJ. Estrogen increases proteasome activity in murine microglial cells. Neurosci Lett 2004;367:60-5.

[35] Hartz AM, Madole EK, Miller DS, Bauer B. Estrogen receptor beta signaling through phosphatase and tensin homolog/phosphoinositide 3-kinase/Akt/glycogen synthase kinase 3 down-regulates blood-brain barrier breast cancer resistance protein. J Pharmacol Exp Ther 2010;334:467-76.

[36] Teoh H, Leung SW, Man RY. Short-term exposure to physiological levels of 17 beta-estradiol enhances endothelium-independent relaxation in porcine coronary artery. Cardiovasc Res 1999;42:224-31.

[37] Li X, Li H, Li XJ. Intracellular degradation of misfolded proteins in polyglutamine neurodegenerative diseases. Brain Res Rev 2008;59:245-52.

[38] Soto C, Estrada LD. Protein misfolding and neurodegeneration. Arch Neurol 2008;65:184-9.

[39] Yamamoto A, Simonsen A. The elimination of accumulated and aggregated proteins: a role for aggrephagy in neurodegeneration. Neurobiol Dis 2011;43:17-28.

[40] Caughey B, Lansbury PT. Protofibrils, pores, fibrils, and neurodegeneration: separating the responsible protein aggregates from the innocent bystanders. Annu Rev Neurosci 2003;26:267-98.

[41] Jellinger KA. Formation and development of Lewy pathology: a critical update. J Neurol 2009;256:270-9.

[42] Ross CA, Poirier MA. Opinion: what is the role of protein aggregation in neurodegeneration? Nat Rev Mol Cell Biol 2005;6:891-8. 
[43] Spires-Jones TL, Stoothoff WH, de Calignon A, Jones PB, Hyman BT. Tau pathophysiology in neurodegeneration: a tangled issue. Trends Neurosci 2009;32:150-9.

[44] Williams AJ, Paulson HL. Polyglutamine neurodegeneration: protein misfolding revisited. Trends Neurosci 2008;31:521-8.

[45] Lin X, Parisiadou L, Gu XL, Wang L, Shim H, Sun L, et al. Leucine-rich repeat kinase 2 regulates the progression of neuropathology induced by Parkinson's-disease-related mutant alpha-synuclein. Neuron 2009;64:807-27.

[46] Xia H, Mao Q Eliason SL, Harper SQ Martins IH, Orr HT, et al. RNAi suppresses polyglutamine-induced neurodegeneration in a model of spinocerebellar ataxia. Nat Med 2004;10:816-20.

[47] Yamamoto A, Lucas JJ, Hen R. Reversal of neuropathology and motor dysfunction in a conditional model of Huntington's disease. Cell 2000;101:57-66.

[48] Alves-Rodrigues A, Gregori L, Figueiredo-Pereira ME. Ubiquitin, cellular inclusions and their role in neurodegeneration. Trends Neurosci 1998;21:516-20.

[49] Liscic RM, Grinberg LT, Zidar J, Gitcho MA, Cairns NJ. ALS and FTLD: two faces of TDP-43 proteinopathy. Eur J Neurol 2008;15:772-80.

[50] Tan CF, Eguchi H, Tagawa A, Onodera O, Iwasaki T, Tsujino A, et al. TDP-43 immunoreactivity in neuronal inclusions in familial amyotrophic lateral sclerosiswith or without SOD1 genemutation. Acta Neuropathol 2007;113:535-42.
[51] Brinton RD, Tran J, Proffitt P, Montoya M. 17 B-Estradiol enhances the outgrowth and survival of neocortical neurons in culture. Neurochem Res 1997;22:1339-51.

[52] Green PS, Simpkins JW. Estrogens and estrogen-like non-feminizing compounds. Their role in the prevention and treatment of Alzheimer's disease. Ann N Y Acad Sci 2000;924: 93-8.

[53] Greenfield JP, Leung LW, Cai D, Kaasik K, Gross RS, Rodriguez-Boulan E, et al. Estrogen lowers Alzheimer ß-amyloid generation by stimulating trans-Golgi network vesicle biogenesis. J Biol Chem 2002;277:12128-36.

[54] Regan RF, Guo Y. Estrogens attenuate neuronal injury due to hemoglobin, chemical hypoxia, and excitatory amino acids in murine cortical cultures. Brain Res 1997;764:133-40.

[55] Dubal DB, Rau SW, Shughrue PJ, Zhu H, Yu J, Cashion AB, et al. Differential modulation of estrogen receptors (ERs) in ischemic brain injury: a role for ERalpha in estradiol-mediated protection against delayed cell death. Endocrinology 2006;147:3076-84.

[56] Joly AL, Wettstein G, Mignot G, Ghiringhelli F, Garrido C. Dual role of heat shock proteins as regulators of apoptosis and innate immunity. J Innate Immun 2010;2:238-47.

[57] Kaushik S, Cuervo AM. Chaperone-mediated autophagy. Methods Mol Biol 2008;445:227-44. 